\title{
Locomotor deficits in recently concussed athletes and matched controls during single and dual-task turning gait: preliminary results
}

\author{
Peter C. Fino ${ }^{1,2^{*}}$, Maury A. Nussbaum ${ }^{3}$ and Per Gunnar Brolinson 2,4
}

\begin{abstract}
Background: There is growing evidence that mild traumatic brain injury (concussion) can affect locomotor characteristics for prolonged periods of time even when physical signs and symptoms are absent. While most locomotor deficits post-concussion have involved straight walking, turning gait has received little attention despite its pervasiveness in everyday locomotion and athletic competition.

Methods: This study longitudinally examined kinematic characteristics during preplanned turning in a small sample of recently concussed athletes $(n=4)$ and healthy matched control athletes $(n=4)$ to examine potential deficits during single and dual-task turning gait over the initial 6 weeks post-injury, with a one-year follow-up. Turning path kinematics (curvature, obstacle clearance, path length), stride kinematics (stride length, stride width, stride time), and inclination angles were calculated from motion capture of participants walking around an obstacle.

Results: Concussed athletes had larger dual-task costs in turning speed and stride time compared to healthy controls. After controlling for speed and turn curvature, recently concussed athletes increased their inclination towards the inside of the turn over time and decreased their stride time compared to controls indicating a prolonged recovery. Kinematic differences between groups were estimated to recover to healthy levels between 100 and 300 days post-injury, suggesting future prospective longitudinal studies should span 6-12 months post-injury.

Conclusion: Turning gait should be included in future studies of concussion and may be a clinically useful tool. Future longitudinal studies should consider examining gait changes for up to 6-12 months post-injury.
\end{abstract}

Keywords: mTBI, Concussion, Brain injury, Gait, Turning, Dual-task

\section{Background}

Sport-related mild traumatic brain injuries, commonly called concussions, affect 1.6-3.8 million people in the United States annually [1]. Within collegiate athletics, concussions account for approximately $5 \%$ of all sportrelated injuries [2], making concussion treatment and rehabilitation an important medical concern. While clinical signs and symptoms typically resolve within 10 days [3] there is growing evidence for an "atypical evolution"

\footnotetext{
*Correspondence: fino@ohsu.edu

'Department of Neurology, Oregon Health \& Science University, 3181 SW Sam Jackson Park Road, L226, Portland, OR 97239, USA

${ }^{2}$ Department of Mechanical Engineering, Virginia Tech, Blacksburg, USA

Full list of author information is available at the end of the article
}

of symptoms [4] with neurological $[5,6]$ and locomotor [7-16] changes that persist beyond two months.

The use of a dual-task (DT) paradigm has been particularly successful at identifying residual deficits postconcussion [17]. During DT gait, simultaneous cognitive and motor tasks compete for limited cortical resources [18] and create gait modifications (dual-task costs) compared to single-task (ST) gait. While such dual-task costs (DTC) are present in healthy young adults [19], larger DTCs have been reported in asymptomatic, recently concussed athletes $[9-13,15,20-24]$. The larger DTCs in recently concussed athletes suggest that a concussion may affect the available cortical resources or shift the prioritization from motor to cognitive tasks $[18,21]$. In 
athletics where gameplay, field, and environmental conditions (e.g., crowd noise) create substantial cognitive and physical loads [25], the complexity-dependent motor differences in medically cleared, recently concussed athletes could influence performance and/or injury risk. However, few studies have examined DTCs with complex non-straight movements to simulate the more dynamic demands of competition.

Some studies have reported greater cognitive DTC (cDTC) during obstacle circumvention [11] and hockeyspecific skating and puck-handling drills [26], but provided little information about the locomotor DTCs (lmDTC), such as changes in gait speed or mediolateral (ML) sway. Similarly, while several studies have used a step-over obstacle as the secondary task to study $\operatorname{lmDTC}$ $[9,27,28]$, few studies have combined a complex motor task with a complex cognitive task to elicit larger lmDTCs [10]. No study to our knowledge has yet examined kinematic changes, with or without a cognitive task, in recently concussed athletes during preplanned turning.

Turning gait is a common [29] complex locomotor task [30-33] applicable to change of direction and reorientation during dynamic athletic movements $[11,16]$. Yet, despite the high risk of slips and falls [34-36], large COM excursions [37], asymmetrical loading [38], and segmental reorientation variability [16] present during turning, the biomechanical $\operatorname{lmDTCs}$ during turning gait in recently concussed athletes remain largely unknown. Due to the high prevalence of turning gait, the segmental reorientation variability in athletes during unplanned turns [16], and the potential correlations to injury risk and performance, both single-task (ST) and DT turning gait in recently concussed athletes present important research areas in concussion management. We hypothesized that ST turning gait would identify kinematic differences between asymptomatic, recently concussed athletes and controls given the increased complexity of turning gait, compared to straight gait, [31-33] and the complexity-dependent effects of concussions on motor performance [10]. The addition of a DT was expected to elicit greater $\operatorname{lmDTC}$ during turning in recently concussed athletes compared to healthy controls and further separate concussed and healthy athletes.

\section{Methods \\ Participants}

Eight NCAA Division I varsity athletes (four concussed, four matched controls: Table 1) participated in this longitudinal study. Concussions were clinically diagnosed by a trained sports medicine physician and referred to this study by their athletic trainer. No concussed athlete had a prior concussion. For each concussed athlete, a control participant was recruited from teammates of the concussed subject and was individually matched based on sport, position, skill level, and stature. Participants with any history of mental illness, diagnosed cognitive impairment, or unresolved acute lower extremity injury were excluded for the study. Controls were excluded if they had suffered any concussion or brain injury within the past year. No control had any prior history of concussion. All participants gave informed written consent, and all recruitment procedures and experimental protocols were approved by the Virginia Tech Institutional Review Board.

\section{Procedures}

Participants were tested at approximately weekly intervals for six weeks, at mean (SD) 7 (0), 16 (2), 23 (2), 29 (1), 37 (2), and 45 (3) days following the concussed participant's concussion. To control for potential musculoskeletal afflictions from recent games or practices that might have influenced gait, each control participant was also tested up to six times and, when possible, on the same days as their competition. Three concussed participants and two control participants also participated in a one-year follow-up testing session occurring at a mean (SD) of 363 (42) days post-concussion. Because of scheduling conflicts, graduations, and transfers, not all participants completed all sessions (Table 1). For the purpose of the current analysis, which examined locomotor changes that remain unresolved after RTP, testing sessions that occurred before the concussed athletes were cleared for full athletic competition were excluded.

Table 1 Descriptive information of all eight participants in matched pairs

\begin{tabular}{lllllll}
\hline ID & Gender & Age (years) & Height $(\mathrm{cm})$ & Mass $(\mathrm{kg})$ & Days Before Full RTP $^{\text {Weeks Tested }^{\mathrm{a}}}$ \\
\hline C1 & Female & 18 & 170 & 63.3 & 7 & $(2,3,4,5,6,52)$ \\
H1 & & 178 & 64.6 & - & $(2,3,4,5,6,52)$ \\
C2 & Male & 20 & 178 & 78.9 & 14 & $(2,3,4,5,6)$ \\
H2 & & 178 & 72.4 & - & $(3,4,5,6)$ \\
C3 & Female & 19 & 163 & 58.7 & 11 & $(3,4,5,6,52)$ \\
H3 & & 163 & 59.0 & - & $(3,4,5,6,52)$ \\
C4 & Female & 18 & 163 & 59.4 & 16 & $(3,4,5,6,52)$ \\
H4 & & 19 & 66.6 & - & $(2,3,4,6)$ \\
\hline
\end{tabular}

Full return-to-play (RTP) was identified as the first day the athlete was medically cleared for full athletic participation in practices and/or games

${ }^{a}$ One year follow-up session represented as 52 weeks post-concussion 
All testing sessions occurred in a basketball gymnasium with minimal distractions and clean hardwood floors. Participants were asked to walk barefoot around an $18 \mathrm{~m} \mathrm{x} 3.5 \mathrm{~m}$ course marked with $1.5 \mathrm{~m}$ tall pylons (2.5 cm diameter PVC pipe) and consisting of several turns of $\sim 90^{\circ}$ (Fig. 1). Participants completed seven laps in each direction around the course. For DT gait, participants were asked to serially subtract by sevens, starting from a random number between 900 and 999, while walking around the course. Participants were not given explicit instruction to prioritize the cognitive or motor task, being instructed only to serially subtract "as fast as you can". For both ST and DT gait, the participants were instructed not to count the number of laps.

Four motion capture cameras (ProReflex MCU 170 120, Qualisys Medical AB, Gothenburg, Sweden) were stationed around one $\sim 90^{\circ}$ turn on the course (Fig. 1) to capture turning kinematics. Reflective spherical markers were placed bilaterally on the calcaneus, with additional markers placed over the xiphoid process and the T9 vertebra. One marker was placed on the pylon to mark its position in space. Data were collected at $120 \mathrm{~Hz}$ and filtered using a phaseless fourth-order Butterworth filter with a $6 \mathrm{~Hz}$ cutoff frequency. A total of 14 turns, seven of both right and left turns, were captured for each condition corresponding to the 14 laps. Short durations with missing markers were spline interpolated. Trials with more than 30 consecutive missing frames $(>0.25 \mathrm{~s})$ were discarded; 30 out of 1120 total trials were discarded.

\section{Analysis}

The upper-body center of mass $\left(\mathrm{COM}_{\mathrm{UB}}\right)$ was estimated using the three-dimensional mean location of the xiphoid process and T9 markers [39]. COM clearance was represented as the minimum horizontal distance from the $\mathrm{COM}_{\mathrm{UB}}$ to the pylon $\left(d_{\text {min }}\right)$. The length of the horizontal trajectory, $L_{\text {path }}$, was computed starting when the athlete proceeded below the pylon in the $y$ direction, and ending when the athlete advanced above the pylon in the $y$ direction (Fig. 2). Quadratic fits to the horizontal trajectory of the $\mathrm{COM}_{\mathrm{UB}}$ were applied to a moving window of three consecutive points. Subsequently, instantaneous $\mathrm{COM}_{\mathrm{UB}}$ curvature in the horizontal plane was calculated from the second derivative of each quadratic polynomial fits.

Heel contacts corresponding to each step were identified using the local minima of the heel marker height. For each turn, a single stride was identified that encompassed the point of maximum curvature and therefore the greatest change in direction. This turning stride was comprised of three heel contacts and it was selected such that the middle heel contact (apex limb) occurred closest to the point of maximum $\mathrm{COM}_{\mathrm{UB}}$ curvature in the $x$ direction (Fig. 2). Stride length (SLength) and stride width (SWidth) were calculated based on the line of progression [40], as shown in Fig. 3, and stride time (STime) was calculated as the time from heel contact to heel contact on the same foot within the turning stride. Stride width was allowed to be negative if the apex limb crossed over the line of progression (Fig. 3).

The strategy used within each turn was characterized using the apex limb. A "step turn" was identified if the apex limb was contralateral to the turn direction (e.g., a right turn with the left leg in stance at the apex), whereas a "spin turn" was identified if the apex limb was ipsilateral to the turn direction (e.g., a right turn with the right leg in stance at the apex) [38]. The mean velocity of the $\mathrm{COM}_{\mathrm{UB}}\left(v_{\text {com }}\right)$ was calculated over the turning stride. The mean curvature, $k$, of the $\mathrm{COM}_{\mathrm{UB}}$ was calculated as the mean of the previously described instantaneous curvature over the turning stride to compare the relationship between velocity and curvature.

\section{Curvature - velocity power law relationship}

A power law relationship between the radius of curvature $(R=1 / k)$ and velocity has previously been defined for turning gait in both outlined [41] and free paths [42]. This power law relationship can be expressed linearly in logarithmic form

$$
\log v_{c o m}(t)=\log A+\alpha \log R(t)
$$

where $R$ is the radius of curvature, $\alpha$ is a constant coefficient, and $A$ is the piecewise velocity gain factor that can

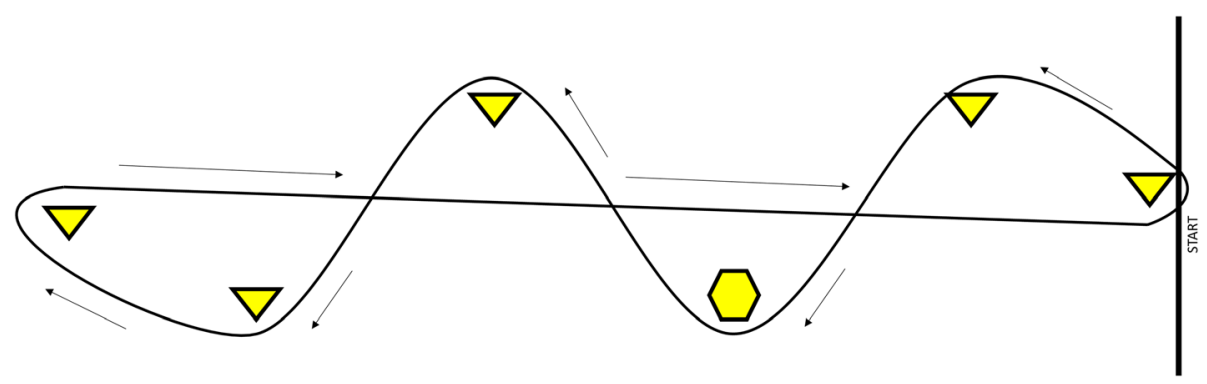

Fig. 1 Depiction of the $18 \mathrm{~m}$ long course. Triangles indicate pylons. The hexagon indicates the pylon recorded with motion capture cameras 


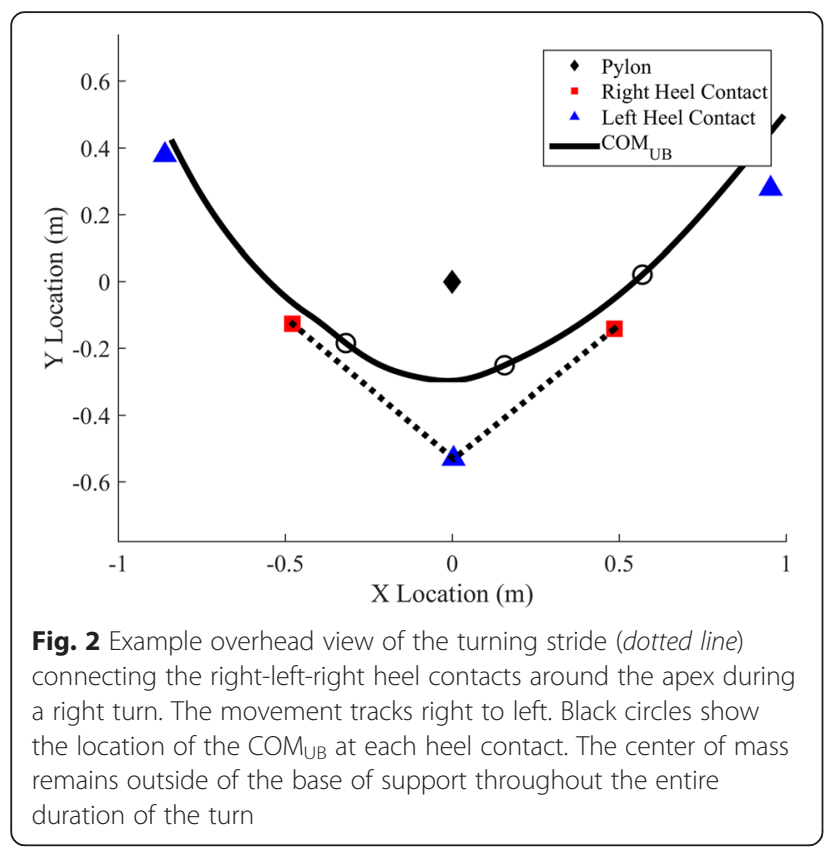

vary with shape [43]. To compare whether the recently concussed athletes exhibited a different relationship between velocity and curvature, the relationships between mean velocities, $v_{\text {com }}$, and corresponding radii of curvature, $1 / k$, were compared for each group using the $\alpha$ and $A$ coefficients determined from linear statistical models.

\section{Mediolateral inclination angles and centripetal force}

The ML inclination angles, $\theta_{1}, \theta_{2}$, and $\theta_{3}$, corresponding to the three heel contacts in each turning stride, were estimated using the frontal plane angle between vertical and the vector connecting the heel to $\mathrm{COM}_{\mathrm{UB}}$ at each heel contact. Because turning is a transient motion, the frontal plane was identified using the vertical plane normal to the instantaneous horizontal velocity of the $\mathrm{COM}_{\mathrm{UB}}$ [44]. Medial inclinations were defined positive (Fig. 4).

The relationship between inclination angle, velocity $v_{\text {com }}$, and curvature $k$ during turning was accounted for using the relation between angle and coefficient of friction, $\mu$ [37]. Using a simple model of a steady state turn of a banked object (mass $m$ ) at an angle of $\theta$ (Fig. 5), balancing centripetal and gravitational moments, $M_{\mathrm{c}}$ and $M_{g}$, about the center of mass

$$
\begin{aligned}
& M_{c}=M_{g} \\
& F_{f} L \cos (\theta)=F_{N} L \sin (\theta) \\
& \mu m g \cos (\theta)=m g \sin (\theta) \\
& \mu=\tan (\theta)
\end{aligned}
$$

and substituting for $\mu$ based on the centripetal force relationship

$$
\begin{aligned}
& F_{f}=m v^{2} \kappa=\mu m g \\
& \mu=\frac{v^{2} \kappa}{g}
\end{aligned}
$$

the inclination angles follow the proportional relationship

$$
\theta \propto \tan ^{-1}\left(v^{2} \kappa\right)
$$

where $g$ is the gravitational constant. Though variability is introduced from the discontinuities of steps during human locomotion and the transient nature of the turns examined here, this proportional relationship was considered when comparing inclination angles.

A total of 10 kinematic measures were analyzed (Table 2). Because many kinematic outcomes depend on the stance limb and gait speed [37], specific lmDTCs for each outcome were not calculated. Instead, ImDTCs
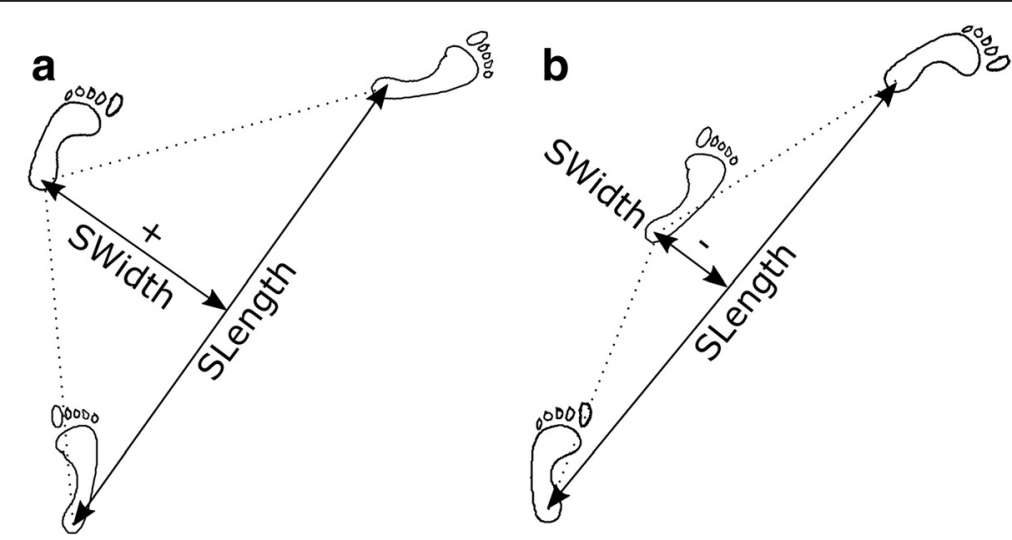

Fig. 3 Illustration of step (a) and spin (b) turns to the right. The stride length, SLength, is the linear distance following the path of progression between successive heel contacts of the same limb. The stride width, SWidth, is the distance, normal to the path of progression, between the apex heel contact and the path of progression 


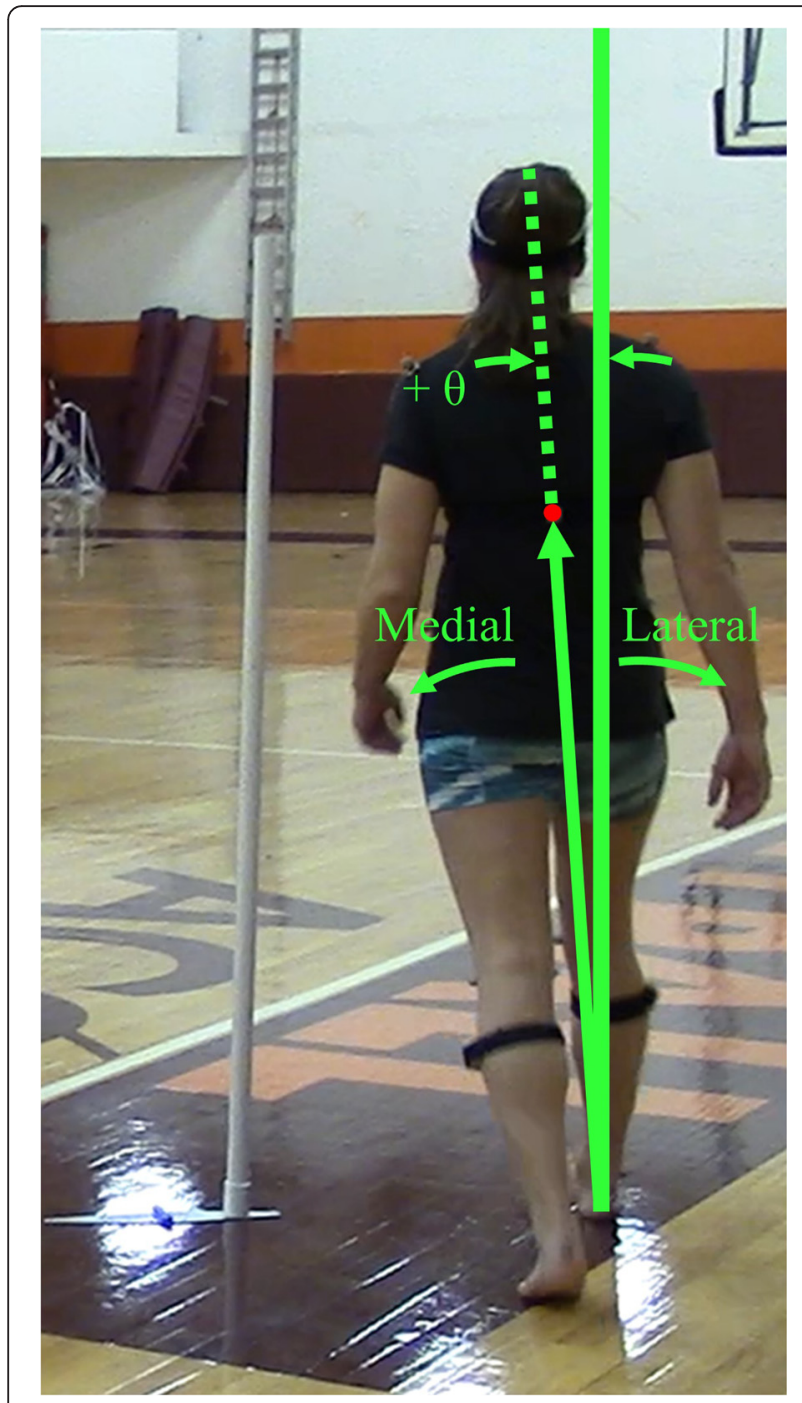

Fig. 4 Depiction of the ML inclination angles, with medial inclinations presented as positive and lateral inclinations presented as negative values, regardless of stance limb

were assessed in the statistical models (see below), with group differences in ImDTC identified by significant group*task interaction effects.

\section{Statistical analysis}

To assess differences between groups, linear mixed models with random intercepts and slopes for time, which account for the within-subject correlations among each participant's trials over time, were fit for each outcome. Given previous findings of decreased gait speed during DT gait and in concussed athletes [10], an initial mixed model was evaluated to compare the influence of group, day, task, as well as all interactions on $v_{\text {com }}$. Subsequent models for $k, L_{\text {path }}$, and $d_{\text {min }}$, included $v_{\text {com }}$ as a covariate, to account for its influence on the kinematic variables during turning [37]. Outcomes of SWidth,

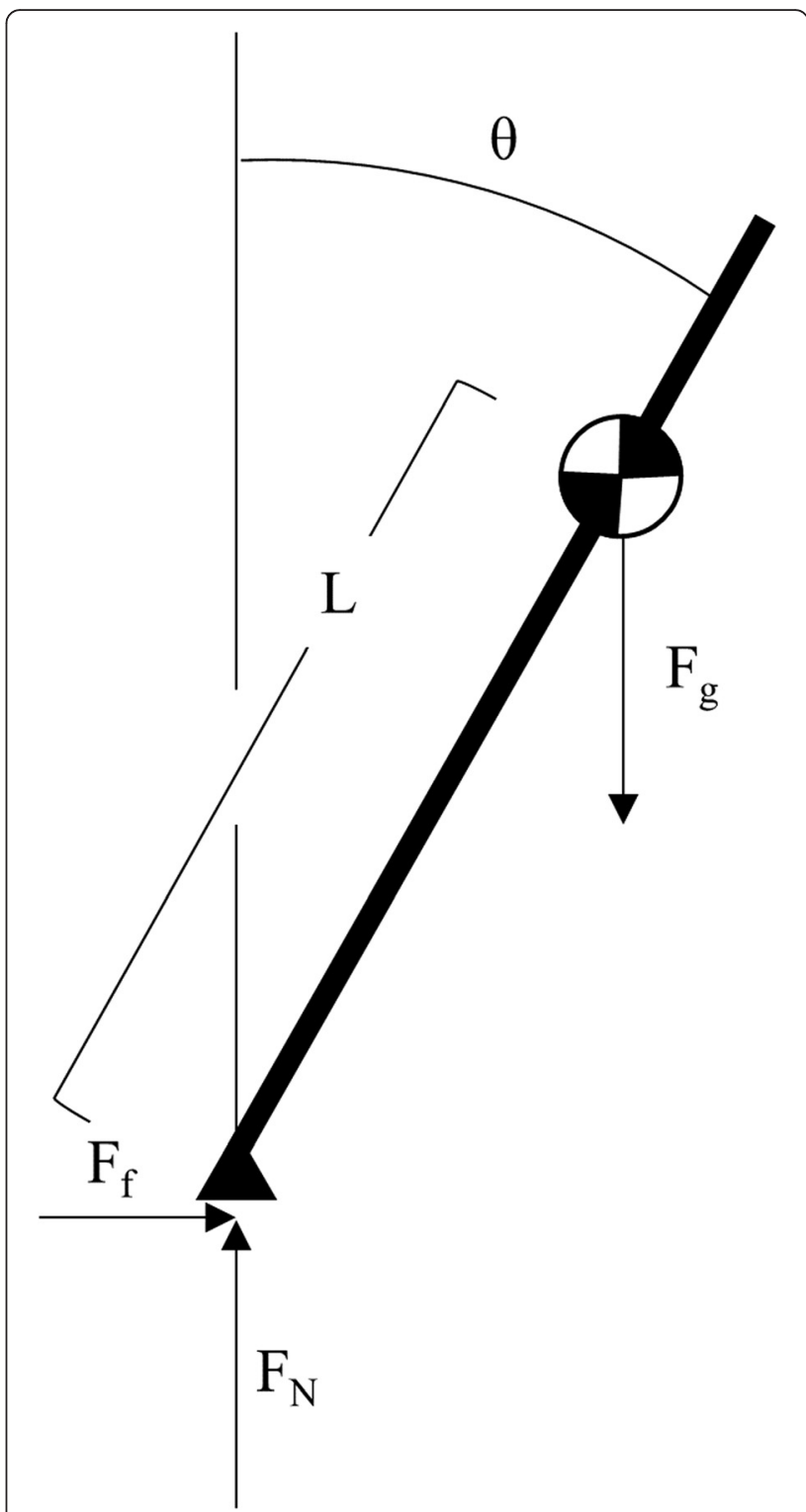

Fig. 5 Simple banked rigid body with inclination angle $\theta$, mass $m$, and length to center-of-mass $L$

SLength, and STime were stratified by turning strategy to account for the kinematic and kinetic differences between step and spin turns [38]. Two-way interactions of group*day, group*task, and day"task, and the three-way group*day*task interaction were included in the initial models. Interaction terms with $p<0.10$ were retained in the final models. Kinematic lmDTC differences between groups were reflected in the group*task or group*day*task interaction.

Power law relationships between $\log v_{\text {com }}$ and $\log 1 / k$ (Eq. 1) were compared between groups using a mixed model including group and a group* $1 / k$ interaction term. The exponent $\alpha$ and coefficient $A$ were determined using the mixed model estimates for each group. Data from 
Table 2 Description of all measured kinematic outcomes

\begin{tabular}{ll}
\hline Outcome & Description \\
\hline$v_{\text {com }}$ & Mean velocity of the COM \\
$k$ & $\begin{array}{l}\text { Mean curvature of horizontal COM trajectory } \\
\text { across turning stride }\end{array}$ \\
$L_{\text {path }}$ & $\begin{array}{l}\text { Total length of COMUB path around the pylon } \\
d_{\text {min }}\end{array}$ \\
SWidth & $\begin{array}{l}\text { Minimum distance from the COM } \\
\text { corner pylon }\end{array}$ \\
SLength & $\begin{array}{l}\text { Distance from apex limb to the line of progression } \\
\text { during the turning stride }\end{array}$ \\
STime & $\begin{array}{l}\text { Linear distance from first the third heel contact } \\
\text { during the turning stride }\end{array}$ \\
$\theta_{1}$ & $\begin{array}{l}\text { Time from first to third heel contact during the } \\
\text { turning stride }\end{array}$ \\
$\theta_{2}$ & $\begin{array}{l}\text { ML inclination angle at the first heel contact of } \\
\text { the turning stride }\end{array}$ \\
$\theta_{3}$ & $\begin{array}{l}\text { ML inclination angle at the second heel contact } \\
\text { of the turning stride } \\
\text { ML inclination angle at the third heel contact of } \\
\text { the turning stride }\end{array}$ \\
\hline
\end{tabular}

concussed one-year follow-ups were excluded from this comparison to prevent potential distortion of the immediate post-concussion relationship.

The inclination angles $\theta_{1}, \theta_{2}$, and $\theta_{3}$ were stratified by turning strategy and compared using mixed models with covariates of group, day, task, and $\tan ^{-1}\left(v_{\text {com }}^{2} k\right)$, following from Eq. 8. Two way interactions of group*day, group*task, day*task, and the three-way interaction of group*day*task were included in initial models. Interactions with $p<0.10$ were retained in the final models.

If significant group*day interaction effects were present in any of the final models, a linear recovery timeline was constructed using the predicted values from the mixed model. The time from injury to full recovery was then estimated using the time when the model estimates of the concussed and control groups crossed. For all models, assumptions were validated using residuals. All statistical analyses were performed in
SAS 9.4 (SAS Institute Inc., Cary, NC, USA) using a two-tailed 0.05 significance level.

\section{Results}

Concussed athletes utilized spin turns at similar frequencies (45\%) to controls (46\%) during both ST and DT turning. A summary of statistical results for $v_{\text {com }}, k$, $L_{\text {path }}, d_{\text {min }}$ is given in Table 3 , with respective longitudinal results in Figs. 6, 7, 8 and 9, respectively. When adjusting for turning velocity, a significant group*day interaction for $k$ was found; the concussed group increased their curvature over time, while controls maintained a constant curvature over time. Path length $\left(L_{\text {path }}\right)$ and COM clearance $\left(d_{\text {min }}\right)$ did not differ between groups when adjusting for turning velocity. The group*task ${ }^{*}$ day interaction was significant for $v_{c o m}$, as the concussed group slowed more than the control group during the DT; the associated ImDTC increased over time (greater difference between ST and DT over time) in the concussed group. Using the mixed model results, the estimated linear recovery time until concussed athletes returned to healthy curvature levels was 398 days.

Longitudinal results for SWidth, SLength, and STime, stratified by strategy, are provided in Figs. 10, 11 and 12. Significant lmDTCs, indicated by significant group*task interactions, were found for STime during both step and spin turns and for SWidth during step turns (Table 4). Significant group*day interactions indicated that the concussed group recovered toward healthy levels over time in SWidth for step turns, STime for step turns, and STime for spin turns. The estimated time until concussed athletes recovered to healthy turning stride characteristics during step turns, assuming a linear recovery, was 391 and 144 days for SWidth and STime, respectively, and 179 days for STime during spin turns. STime increased during the DT condition regardless of strategy. Though SLength increased over time (step turns) and decreased during DT turning (spin turns), no differences in SLength were found between groups.

Table 3 Model parameters and inference values from the final linear mixed models for $v_{\text {com, }}, L_{\text {path }}$ and $d_{\text {min }}$

\begin{tabular}{|c|c|c|c|c|c|c|c|c|}
\hline & \multicolumn{2}{|l|}{$\underline{V_{\text {com }}}$} & \multicolumn{2}{|l|}{ k } & \multicolumn{2}{|l|}{$L_{\text {path }}$} & \multicolumn{2}{|l|}{$d_{\min }$} \\
\hline & $\overline{\beta(S E) * 10^{3}}$ & $p$ value & $\overline{\beta(\mathrm{SE}) * 10^{3}}$ & $p$ value & $\overline{\beta(S E) * 10^{3}}$ & $p$ value & $\overline{\beta(\mathrm{SE}) * 10^{3}}$ & $p$ value \\
\hline Group (G) & $-70.0(79.5)$ & 0.379 & $-139.2(90.5)$ & 0.124 & $105.2(130.6)$ & 0.421 & $11.1(37.9)$ & 0.770 \\
\hline Day (D) & $0.80(0.62)$ & 0.243 & $0.05(0.13)$ & 0.736 & $-0.28(0.07)$ & 0.004 & $0.04(0.04)$ & 0.394 \\
\hline Task Condition (T) & $-28.3(6.86)$ & $<0.0001$ & $-22.9(10.5)$ & 0.030 & $11.4(9.6)$ & 0.234 & $-11.1(3.47)$ & 0.183 \\
\hline$v_{\text {com }}$ & - & - & $-1292(73.3)$ & $<0.0001$ & $876.5(66.1)$ & $<0.0001$ & $-32.4(24.3)$ & 0.001 \\
\hline$G \times D$ & $0.33(0.86)$ & 0.703 & $0.35(0.17)$ & 0.036 & - & - & - & - \\
\hline$G \times T$ & $-17.8(9.51)$ & 0.061 & - & - & - & - & - & - \\
\hline$D \times T$ & $-0.07(0.05)$ & 0.198 & - & - & - & - & - & - \\
\hline$G \times D \times T$ & $-0.21(0.07)$ & 0.003 & - & - & - & - & - & - \\
\hline
\end{tabular}

Beta coefficients and standard errors (SE) are shown multiplied by $10^{3}$. Higher-order interactions terms with $p<0.10$ were retained in the final model. Significant terms are highlighted in bold 


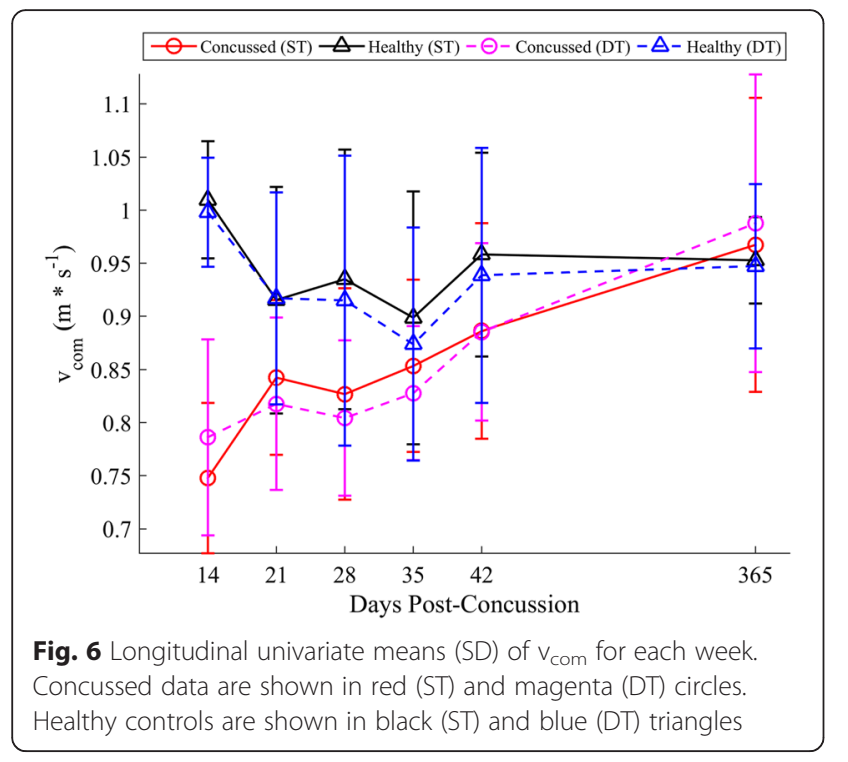

Linear fits between $\log v_{c o m}$ and $\log 1 / k$ yielded estimated mean (SE) of $-0.24(0.06)$ for parameter $A$ in concussed athletes within 6 weeks of their concussion, and $-0.08(0.04)$ in controls (Fig. 13). The difference between groups was significant $(p=0.009)$, indicating that $A$ significantly decreased in concussed athletes during the first 6 weeks. No significant interaction was found, indicating that $\alpha$ was similar between concussed and controls, whose respective values were $0.24(0.03)$ and $0.21(0.02)$.

Longitudinal results for each angle are provided in Figs. 14, 15 and 16. When adjusting for the $\tan ^{-1}\left(v_{c o m}^{2} k\right)$ term (Table 5), significant group*day interactions were found for $\theta_{1}$ during spin turns and $\theta_{2}$ for both strategies, indicating that the concussed group increased the magnitude of inclination with increasing time post-injury.

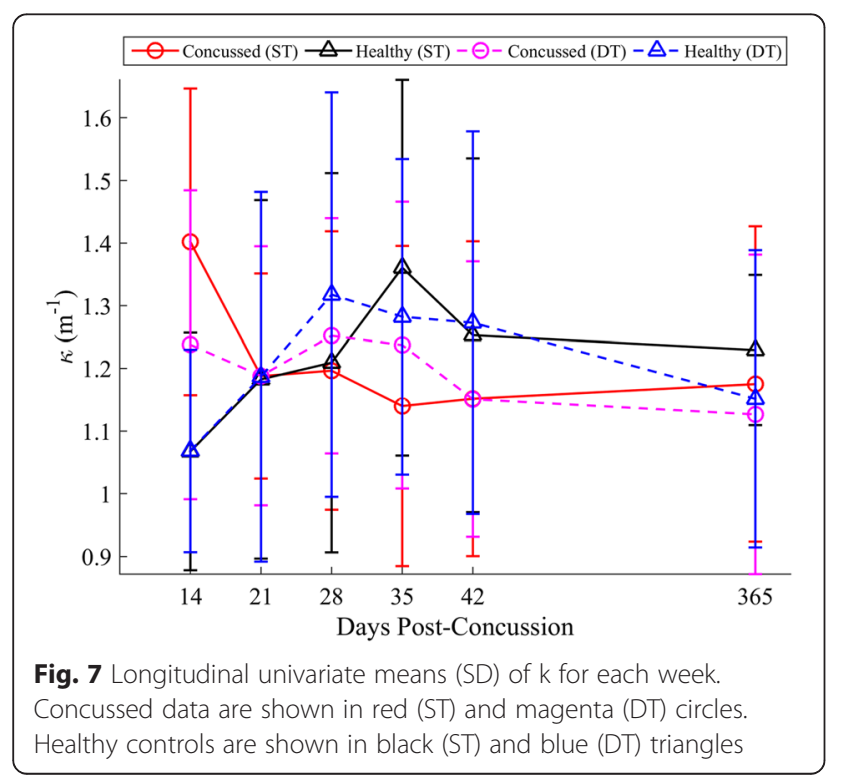

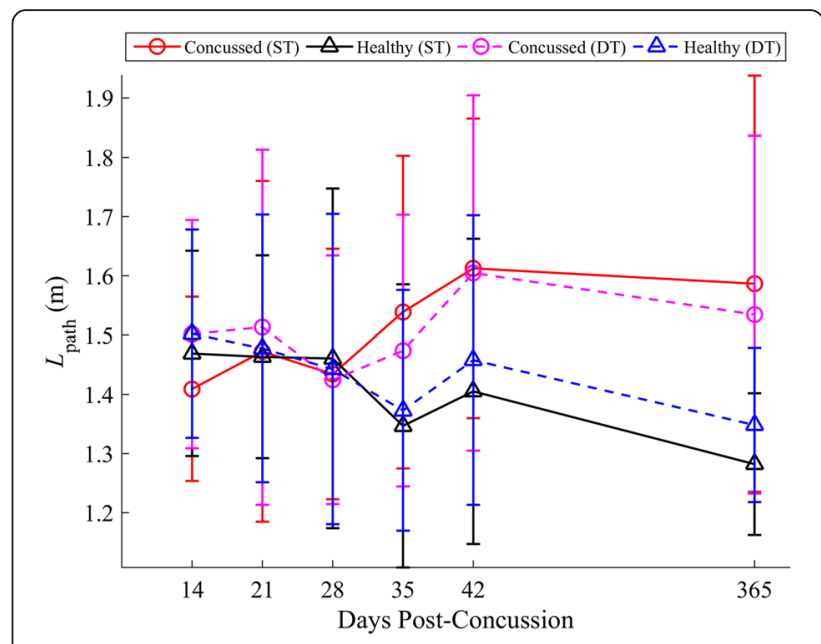

Fig. 8 Longitudinal univariate means (SD) of $L_{\text {path }}$ for each week. Concussed data are shown in red (ST) and magenta (DT) circles. Healthy controls are shown in black (ST) and blue (DT) triangles

For ipsilateral stance limbs during the second half of the turning stride ( $\theta_{3}$ step turns), a group*day"task interaction was found, indicating an increase in the $\operatorname{lmDTC}$ difference between concussed and control groups as time increased. The estimated linear recovery time for concussed athletes was 115 and 123 days during step turns for $\theta_{2}$ and $\theta_{3}$, respectively, 239 days for $\theta_{1}$ during spin turns, and 222 days for $\theta_{2}$ during spin turns. As the DTC differences increased for $\theta_{3}$ over time, no recovery date could be estimated.

\section{Discussion}

This study prospectively examined single- and dual-task turning kinematics in recently concussed and healthy

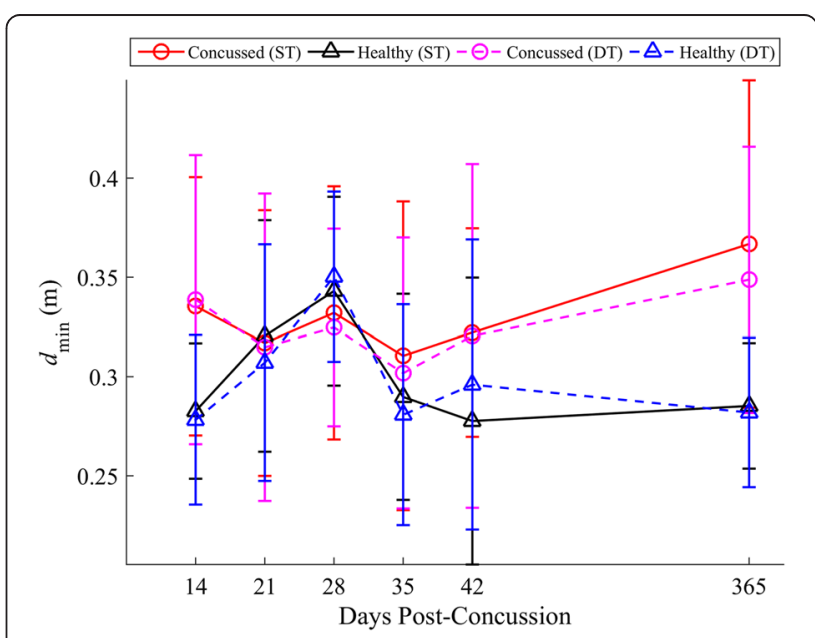

Fig. 9 Longitudinal univariate means (SD) of $d_{\min }$ for each week. Concussed data are shown in red (ST) and magenta (DT) circles. Healthy controls are shown in black (ST) and blue (DT) triangles 


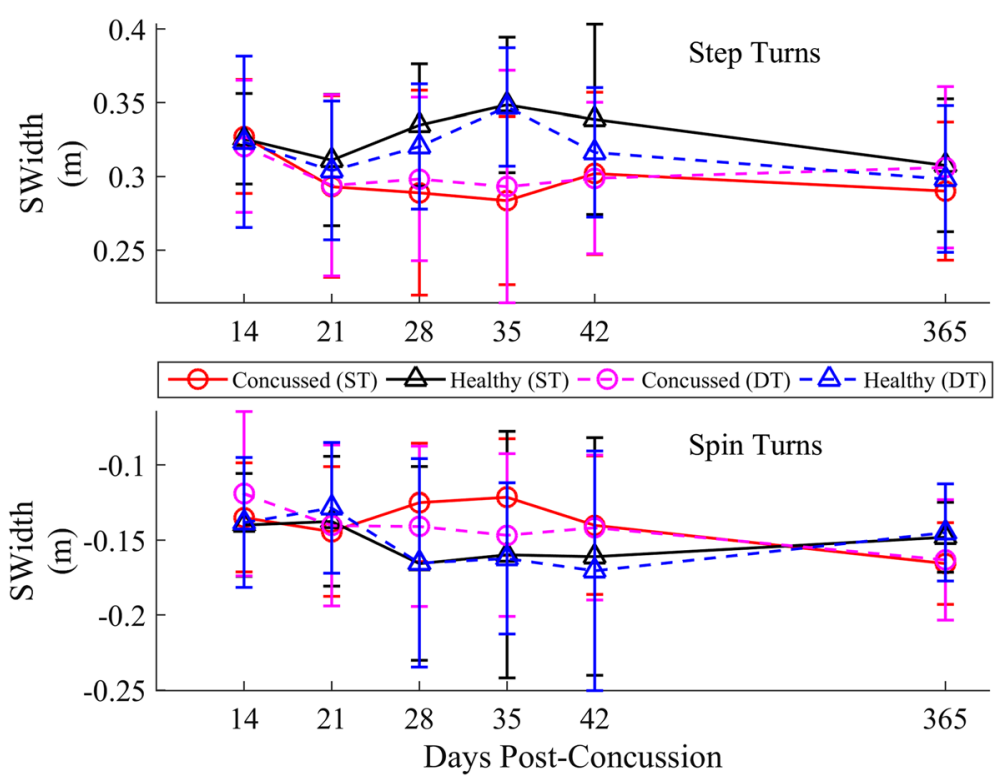

Fig. 10 Longitudinal univariate means (.SD) of stride width, SWidth for each week stratified by step (top) and spin (bottom) turns. Concussed data are shown in red (ST) and magenta (DT) circles. Healthy controls are shown in black (ST) and blue (DT) triangles

control athletes. It provides the first preliminary evidence of kinematic differences during ST and DT preplanned turning gait in a small sample of recently concussed athletes. Despite all athletes being medically cleared for competition, concussed athletes showed greater lmDTCs in SWidth, and STime compared to healthy controls. Additionally, several outcomes - including SWidth, STime, ML inclination angles $\left(\theta_{1}, \theta_{2}\right.$, and $\theta_{3}$ ) and curvature - showed different patterns over time between groups when adjusted for turning speed, which suggests that turning gait may recover slower than other symptoms. Overall, these differences between groups over time describe the effects of concussion on turning gait in three domains: path trajectory $(k)$, stride characteristics (SWidth, SLength, STime), and body orientation $\left(\theta_{1}, \theta_{2}, \theta_{3}\right)$.
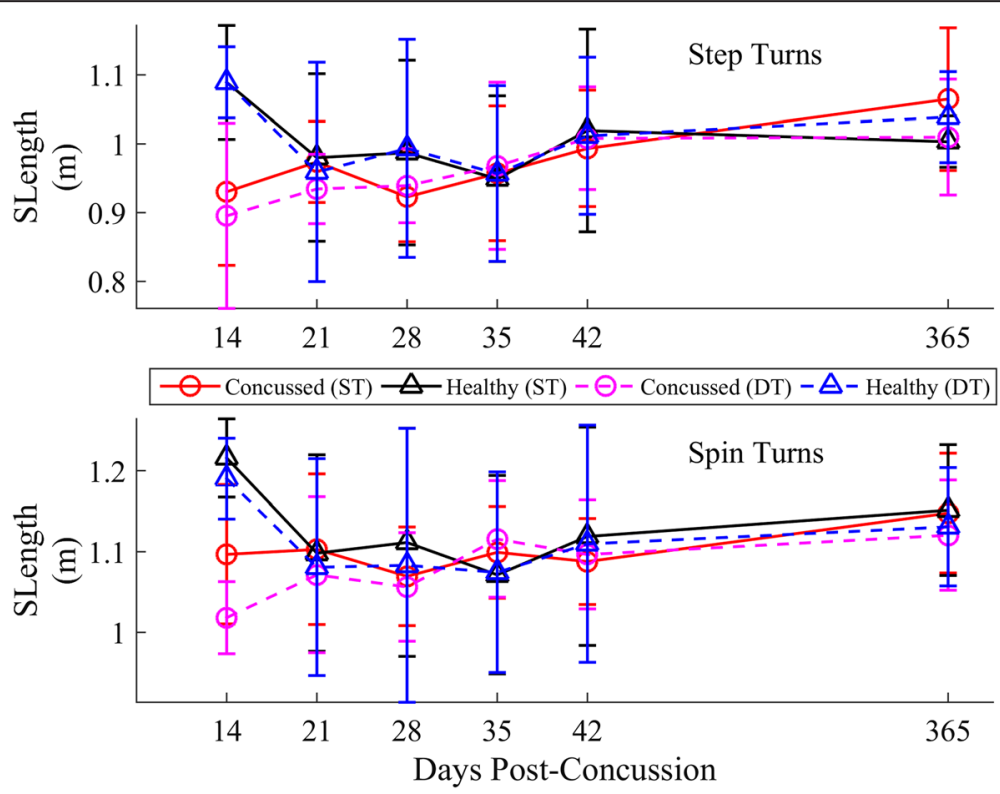

Fig. 11 Longitudinal univariate means (SD) of stride length, SLength for each week stratified by step (top) and spin (bottom) turns. Concussed data are shown in red (ST) and magenta (DT) circles. Healthy controls are shown in black (ST) and blue (DT) triangles 


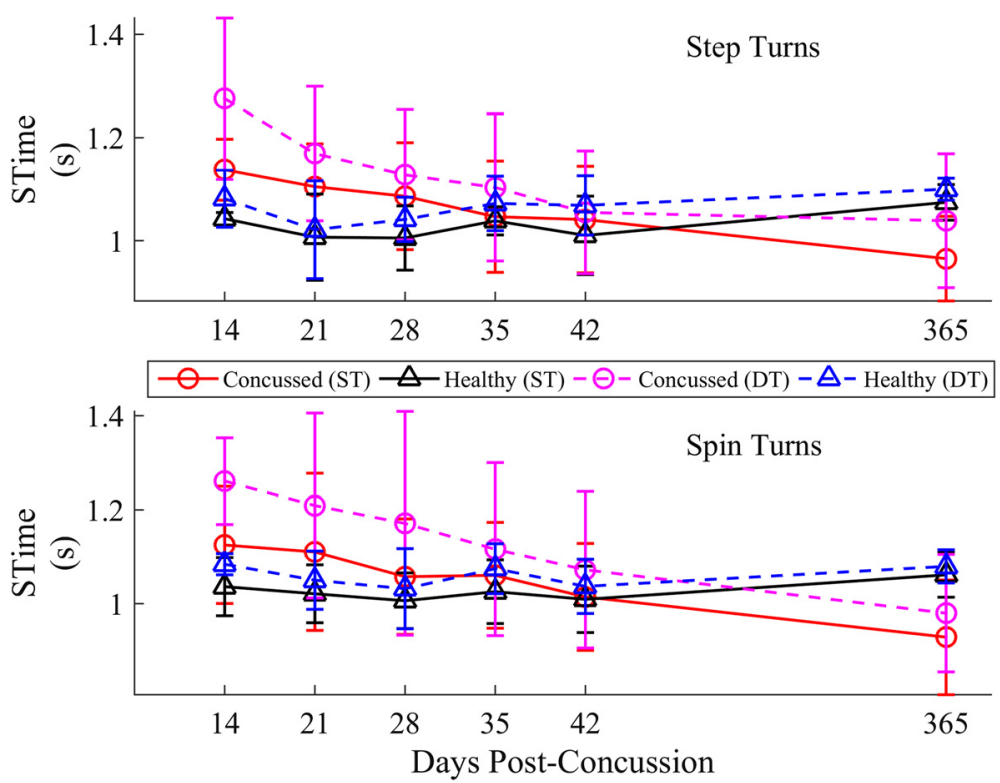

Fig. 12 Longitudinal univariate means (SD) of stride time, STime for each week stratified by step (top) and spin (bottom) turns. Concussed data are shown in red (ST) and magenta (DT) circles. Healthy controls are shown in black (ST) and blue (DT) triangles

\section{Path trajectory}

One key finding of this preliminary study is a decreased velocity gain factor $(A)$ in concussed athletes relative to healthy controls. While both groups exhibited similar power law relationships between $v_{\text {com }}$ and $1 / k$, the concussed group walked with slower velocities across the range of curvatures. Similar modulations of $A$ have been reported as individuals transition to different outlined trajectories [41, 43], but only when the path is predefined. Though individuals follow stereotyped behavior without a predefined path [45], Olivier and Cretual [42] argued that the lack of a consistent power relationship over the course of one turn indicated that a simple turn is not performed by first planning the trajectory and then by following the planned path. Instead, the motor control system is simplified by coupling velocity and curvature and minimizing the deviations in an orientation/velocity phase space from one trajectory to the next [42]. We found similar $\alpha$ values between the concussed and control groups, suggesting that this long term coupling remains present post-concussion. However, the lower $A$ values post-concussion indicate that the relationship has

Table 4 Model parameters and inference values from the final linear mixed models for SWidth, SLength, and STime

\begin{tabular}{|c|c|c|c|c|c|c|}
\hline & \multicolumn{2}{|l|}{ SWidth } & \multicolumn{2}{|l|}{ SLength } & \multicolumn{2}{|l|}{ STime } \\
\hline & $\overline{\beta(S E) * 10^{3}}$ & $\overline{p \text { value }}$ & $\overline{\beta(S E) * 10^{3}}$ & $\overline{p \text { value }}$ & $\overline{\beta(S E) * 10^{3}}$ & $\overline{p \text { value }}$ \\
\hline \multicolumn{7}{|c|}{ Step $(n=593)$} \\
\hline G & $-42.9(33.5)$ & 0.201 & $-31.1(71.6)$ & 0.664 & $51.5(57.8)$ & 0.373 \\
\hline $\mathrm{D}$ & $-0.05(0.02)$ & 0.088 & $0.27(0.10)$ & 0.035 & $0.11(0.07)$ & 0.193 \\
\hline $\mathrm{T}$ & $-7.7(4.3)$ & 0.076 & $-6.7(6.3)$ & 0.296 & $33.1(9.1)$ & 0.0003 \\
\hline$G \times D$ & $0.11(0.03)$ & 0.0002 & - & - & $-0.36(0.10)$ & 0.0002 \\
\hline$G \times T$ & $12.5(5.9)$ & 0.034 & - & - & $37.5(12.3)$ & 0.003 \\
\hline \multicolumn{7}{|c|}{ Spin $(n=497)$} \\
\hline G & $15.6(26.6)$ & 0.556 & $-29.5(77.0)$ & 0.702 & $67.8(73.2)$ & 0.355 \\
\hline $\mathrm{D}$ & $-0.06(0.02)$ & 0.012 & $0.47(0.23)$ & 0.085 & $0.07(0.07)$ & 0.341 \\
\hline $\mathrm{T}$ & $-1.5(3.9)$ & 0.701 & $-18.0(5.6)$ & 0.001 & $32.2(11.8)$ & 0.007 \\
\hline$G \times D$ & - & - & - & - & $-0.38(0.09)$ & $<0.0001$ \\
\hline$G \times T$ & - & - & - & - & $38.5(16.4)$ & 0.023 \\
\hline
\end{tabular}

Beta coefficients and standard errors (SE) are shown multiplied by $10^{3}$. Higher-order interactions terms with $p<0.10$ were retained in the final model. Each outcome was stratified by turning strategy. Significant terms are highlighted in bold 


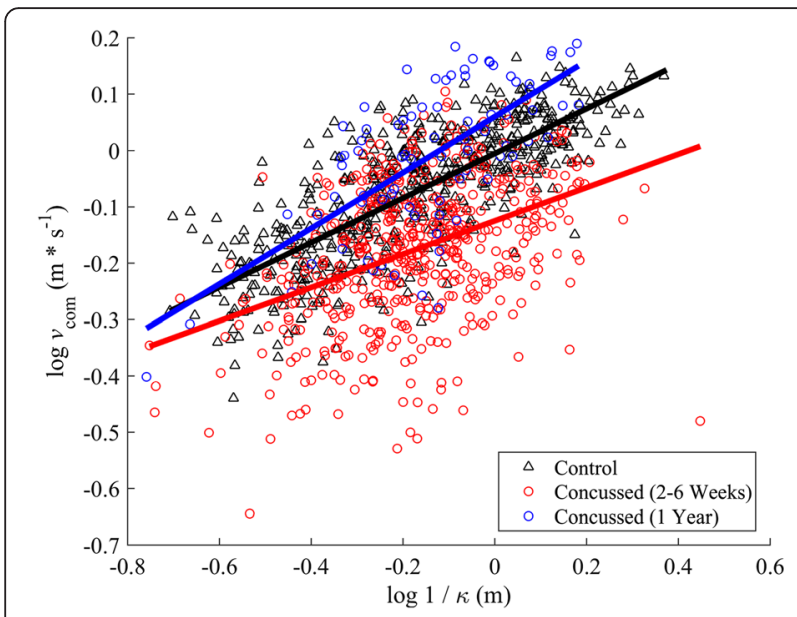

Fig. 13 Linear fits of the power law relationship between velocity and radius of curvature for controls (black, $R^{2}=0.59$ ), concussed athletes within 6 weeks on injury (red, $R^{2}=0.18$ ) and concussed athletes after one year (blue, $R^{2}=0.45$ ). No difference in slopes were found between the fits, but a significant decrease in the velocity gain function A in concussed athletes 2-6 weeks post-concussion was found

shifted to a slower speed, consistent with previously described decreases in gait speed post-concussion [10]. Given the similar values of $\alpha$ between groups, though, it is possible that some of the navigational deficits reported in concussed individuals [11] are predominantly changes in gait speed and not differences in an intrinsic velocity curvature relationship that may affect path characteristics. We have previously reported differences in straight gait speed in this sample population [46] matching previously reported data [15]. Turning speed may also be a useful indicator of recovery based on the present results.

Our results do not indicate whether the path selection was quantitatively different between groups, but trajectories between groups and conditions were qualitatively similar (Fig. 17) and there were no significant differences in path length $\left(L_{\text {path }}\right)$ or minimum clearance $\left(d_{\text {min }}\right)$. The present results, as evidenced by low $R^{2}$ values from the velocity-curvature relationship (Fig. 12) in concussed compared to healthy athletes, do agree with Baker and Cinelli [7] that concussed athletes are more variable in their path selection. It is likely that, on average, concussed athletes can exhibit healthy stereotyped path selection $[45$, 47] but do so with greater variability, similar to their increased joint-coordination [27] and step variability [14].

We did not find a significant difference between groups in minimum clearance $\left(d_{\text {min }}\right)$, though overall conclusions are limited due to the small sample size. However, this appears to contradict Fait et al. [11], who described greater minimum clearances in athletes post-concussion compared to controls. Upon further investigation, however, the apparent differences in clearance reported by Fait et al. [11] may have been due to slightly slower gait speeds in the concussed group. Results presented here and by Fino et al. [37] showed a strong inverse relationship between walking velocity and the minimum obstacle clearance. Additionally, Fait et al. [11] utilized an in-line obstacle circumvention paradigm, where the goal remained fixed and an obstacle was placed in the direct path to that goal. Conversely, we used an obstacle navigation task, where participants were instructed to pass on a specific side of each obstacle in
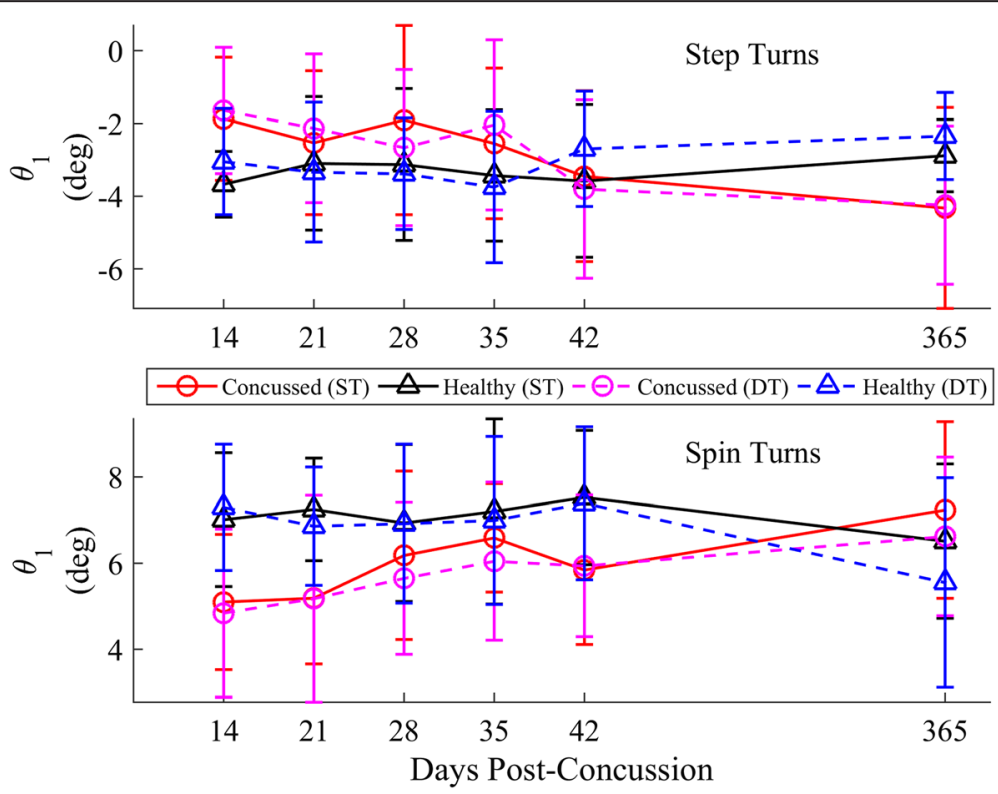

Fig. 14 Longitudinal univariate means (SD) of ML inclination angle $\theta_{1}$ for each week stratified by step (top) and spin (bottom) turns. Concussed data are shown in red (ST) and magenta (DT) circles. Healthy controls are shown in black (ST) and blue (DT) triangles 


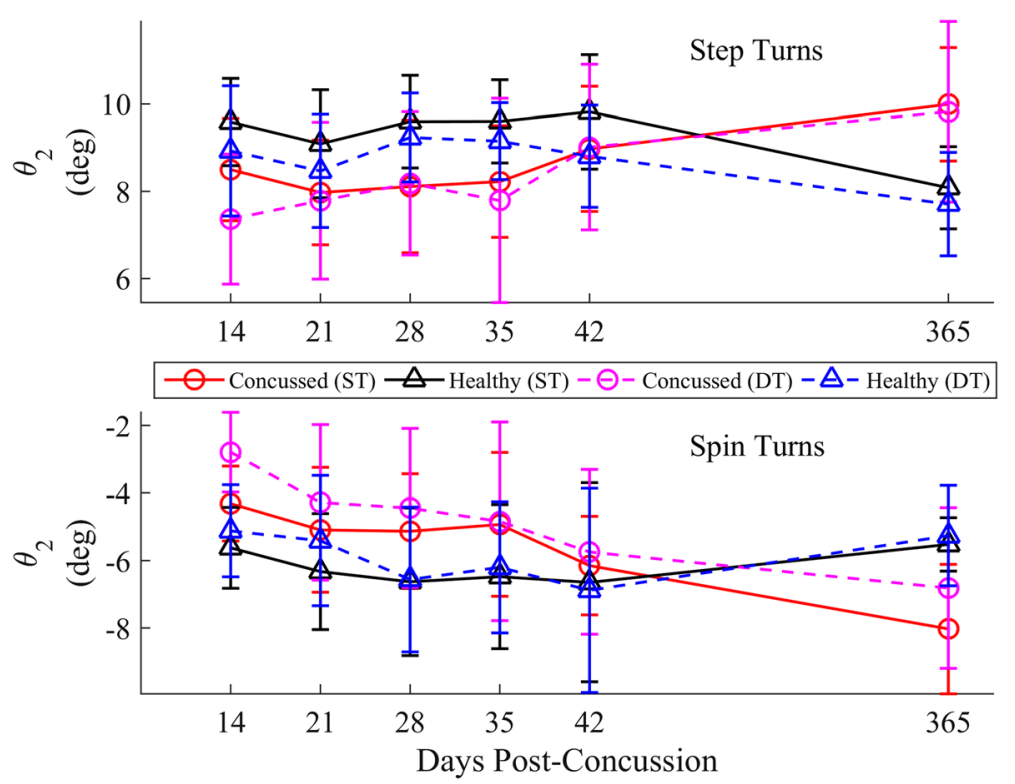

Fig. 15 Longitudinal univariate means (SD) of ML inclination angle $\theta_{2}$ for each week stratified by step (top) and spin (bottom) turns. Concussed data are shown in red (ST) and magenta (DT) circles. Healthy controls are shown in black (ST) and blue (DT) triangles

route to the next. It is possible these different tasks elicited distinct behaviors [48] considering that the required change in heading angle was much larger in the current study.

\section{Stride characteristics}

Concussed athletes exhibited consistent $\operatorname{lmDTC}$ increases in SWidth and STime, widening and slowing their strides during the DT more than the control group. This greater slowing of strides during DT concussed gait has previously been described in straight walking $[15,49]$, but no prior work (to our knowledge) has described this result during turning gait. Larger DTCs in recently concussed athletes are often attributed to a diminished multi-tasking capacity post-concussion, suggesting that a concussion may affect the available

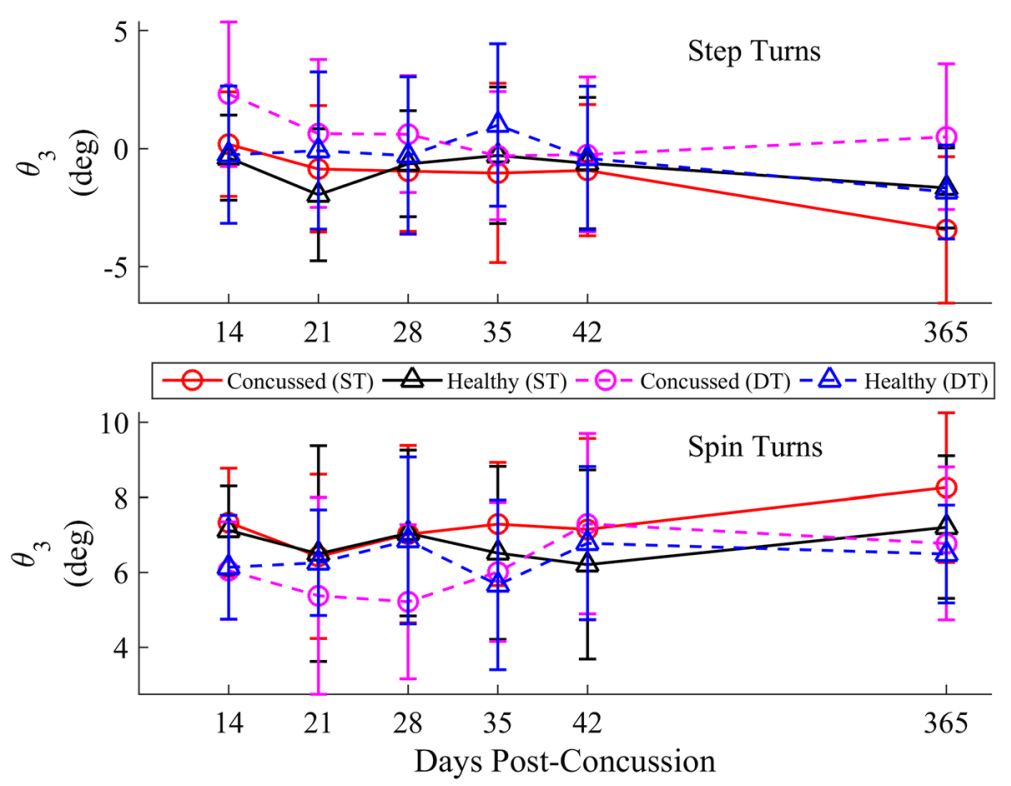

Fig. 16 Longitudinal univariate means (SD) of ML inclination angle $\theta_{3}$ for each week stratified by step (top) and spin (bottom) turns. Concussed data are shown in red (ST) and magenta (DT) circles. Healthy controls are shown in black (ST) and blue (DT) triangles 
Table 5 Model parameters and inference values from the final linear mixed models for the three ML inclination angles, $\theta_{1}, \theta_{2}$, and $\theta_{3}$

\begin{tabular}{|c|c|c|c|c|c|c|}
\hline & \multicolumn{2}{|l|}{$\theta_{1}$} & \multicolumn{2}{|l|}{$\theta_{2}$} & \multicolumn{2}{|l|}{$\theta_{3}$} \\
\hline & $\overline{\beta(S E) * 10^{3}}$ & $p$ value & $\overline{\beta(\mathrm{SE}) * 10^{3}}$ & $p$ value & $\overline{\beta(\mathrm{SE}) * 10^{3}}$ & $p$ value \\
\hline \multicolumn{7}{|l|}{ Step $(n=593)$} \\
\hline G & $151.0(653.6)$ & 0.817 & $-677.7(623.6)$ & 0.278 & $806.0(1056)$ & 0.446 \\
\hline D & $-0.09(0.74)$ & 0.904 & $-2.2(1.8)$ & 0.275 & $-3.2(2.5)$ & 0.236 \\
\hline $\tan ^{-1}\left(V_{\mathrm{com}}^{2} k\right)$ & $-7241(899.0)$ & $<0.0001$ & $4605(565.3)$ & $<0.0001$ & $6754(1441)$ & $<0.0001$ \\
\hline $\mathrm{T}$ & $-94.6(149.4)$ & 0.527 & $-291.2(90.0)$ & 0.001 & 1198 (394.9) & 0.003 \\
\hline$G \times D$ & - & - & $5.9(2.4)$ & 0.015 & $-6.6(3.2)$ & 0.042 \\
\hline$G \times T$ & - & - & - & - & $203.0(542.1)$ & 0.708 \\
\hline $\mathrm{D} \times \mathrm{T}$ & - & - & - & - & $-3.1(3.0)$ & 0.295 \\
\hline$G \times D \times T$ & - & - & - & - & $9.8(4.0)$ & 0.016 \\
\hline \multicolumn{7}{|l|}{ Spin $(n=497)$} \\
\hline G & $-1338(456.2)$ & 0.004 & 1107 (1241) & 0.373 & $443.5(474.8)$ & 0.351 \\
\hline $\mathrm{D}$ & $-2.7(1.7)$ & 0.172 & $-0.53(1.33)$ & 0.704 & $0.24(0.81)$ & 0.775 \\
\hline $\tan ^{-1}\left(V_{c o m}^{2} k\right)$ & 3738 (1009) & 0.0002 & $-5344(769.4)$ & $<0.0001$ & 7490 (1109) & $<0.0001$ \\
\hline $\mathrm{T}$ & $-77.4(157.9)$ & 0.625 & $356.2(194.6)$ & 0.068 & $-412.1(177.0)$ & 0.023 \\
\hline$G \times D$ & $5.6(2.3)$ & 0.016 & $-5.0(1.8)$ & 0.005 & - & - \\
\hline$G \times T$ & - & - & $-222.5(272.1)$ & 0.414 & - & - \\
\hline$D \times T$ & - & - & $-1.4(1.5)$ & 0.370 & - & - \\
\hline$G \times D \times T$ & - & - & $4.0(2.1)$ & 0.054 & - & - \\
\hline
\end{tabular}

Beta coefficients and standard errors (SE) are shown multiplied by $10^{3}$. Higher order interactions terms with $p<0.10$ were retained in the final model. Outcomes were stratified by turning strategy. Significant terms are highlighted in bold

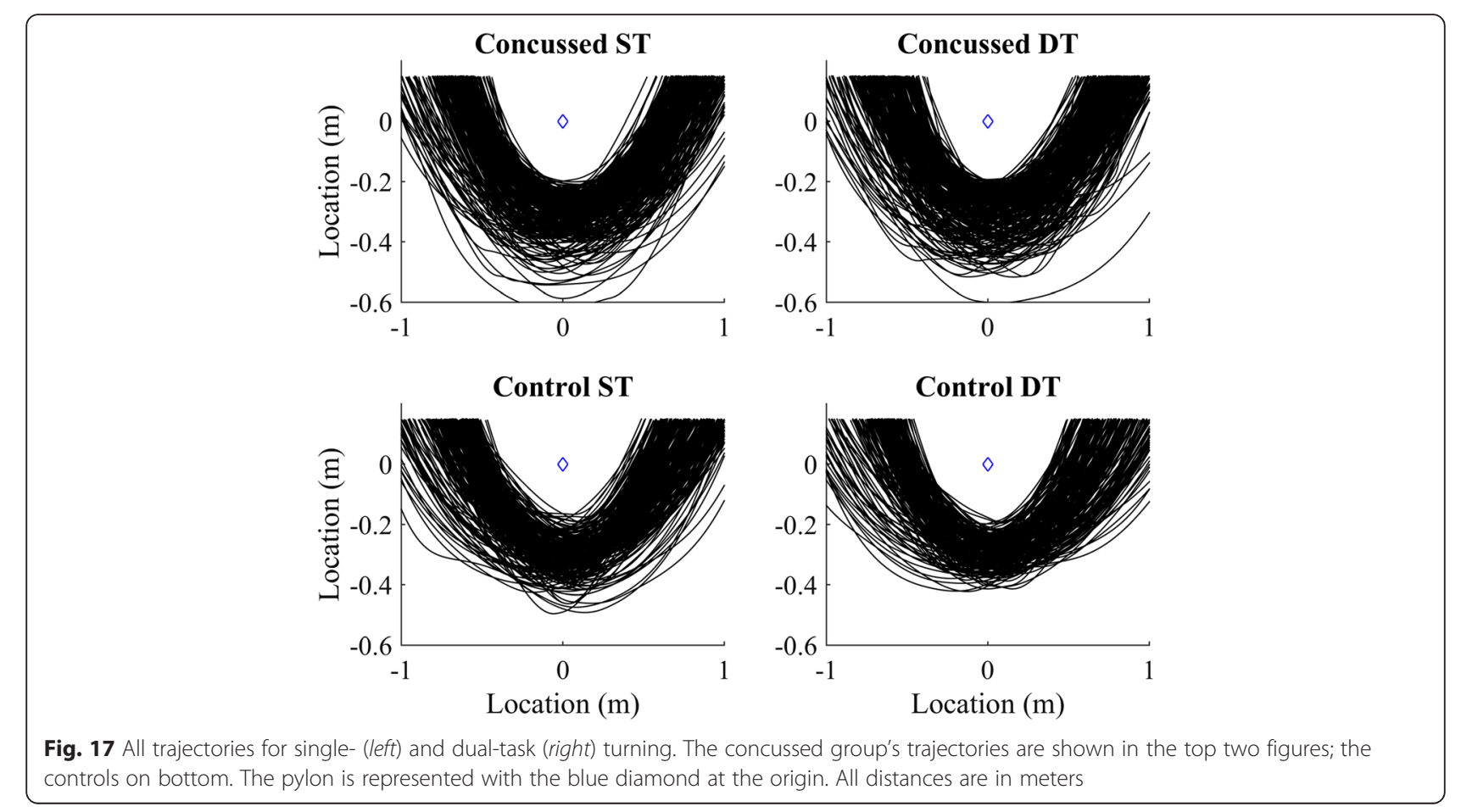


cortical resources or shift the prioritization from motor to cognitive tasks $[18,21]$.

We did not find differences in stride length between groups. Previous studies have reported a decrease or no change in stride length in recently concussed athletes during DT straight walking $[23,49,50]$. Based on the longer STimes in the concussed group and similar SLengths between groups, the differences in gait speed between groups were likely controlled by increasing the STime and not by shortening the SLength during turning.

\section{Body orientation}

Recently concussed athletes increased their inclination towards the turn over time, nearing the inclination angles used by controls, after adjusting for the $\tan ^{-1}\left(v_{c o m}^{2} k\right)$. Based on a simplistic model of a continuous rolling rigid body, the inclination angle should be directly proportional to $\tan ^{-1}\left(v_{c o m}^{2} k\right)$. Given that the concussed athletes generally walked slower across similar curvatures, lower magnitudes of $\theta$ in concussed athletes were expected. However, this group difference was present, though it resolved over time, even when adjusting the model for tan ${ }^{-1}\left(v_{c o m}^{2} k\right)$. For a given velocity and curvature, concussed athletes exhibited less inclination towards the inside of the turn (less medial inclination during step turns, less lateral inclination during spin turns). One may interpret this decreased inclination as another cautious adaptation of the concussed group in order to preserve stability [13, 21,49 ] and maintain the COM closer to the base-ofsupport (BOS). Yet, concussed individuals exhibit greater ML sway during straight walking and obstacle crossing $[12,20,23,28,49]$. At this time, it is unclear why this paradoxical change occurs between curved and straight walking.

While only limited kinematic data were collected in this preliminary study, the geometric relationship between inclination angle and centripetal force can be used as an initial step to examine potential kinetic differences between concussed and control athletes during turning. Using the simple banked rigid body model (Fig. 5), a change in $\theta$ introduces a new coronal plane moment if velocity, curvature, and mass are held constant (based on Eq. 3). For a given curvature and velocity, a decrease in $\theta$ induces a moment counteracting the moment from centripetal force acting on the COM; an increase in $\theta$ induces a moment acting with the centripetal force moment. Interestingly, similar placement of the COM closer towards the stance limb during cutting, accomplished through lateral trunk flexion, has been associated with ACL injuries [51], illustrating the potential risk of decreased inclination during sport-related maneuvers. The current preliminary investigation was not designed to investigate how, where (ankle, knee, hip), or if these increased moments manifest. Yet, these initial results encourage future research examining potential kinetic differences during fast-paced sport-related turning and cutting following concussion, which may be a contributing factor to the increased rate of acute musculoskeletal injuries post-concussion [52, 53].

\section{Timeline of effects and recovery}

Notably, all data presented here were collected after the concussed athletes had returned to full athletic participation. Thus, these results support the growing body of literature indicating locomotor capacity has a prolonged recovery compared to clinical symptoms $[7-13,15,16$, 20-24, 26]. Despite the absence of clinical signs or symptoms, gait deficits post-concussion persisted, and it is unclear if or when any such deficits resolve. Several locomotor abnormalities have been found to persist at least 30 days post-concussion $[7,11,13,14,26]$, with some deficits lasting beyond 60 days [12]. Our results agree with this earlier evidence, by predicting that the recovery of several kinematic abnormalities persist beyond six weeks post-concussion. Yet, we are not aware of any previous study that has prospectively examined recovery beyond 2 months to investigate a full recovery timeline. Approximations based on group*day interaction terms in the present statistical models suggest that complete recovery may not occur until after 200300 days post-concussion. These linear approximations should be interpreted cautiously, though, given the likelihood of a non-linear recovery pattern, the reliance on only mean estimates, the small sample size, and the general lack of data immediately surrounding the estimated recovery time. However, the results do give guidance for the design of future prospective longitudinal studies examining locomotor capabilities post-concussion, suggesting the need to follow participants for 6-12 months post-concussion. Interestingly, these estimates do fit within the same timeframe of reported increases in musculoskeletal and subsequent injuries post-concussion [52-54], reinforcing the need for comprehensive 6-12 month longitudinal studies.

\section{Limitations}

The small sample size of this preliminary study is a primary limitation and prompts caution when generalizing these results to an entire population. Nevertheless, significantly different $\operatorname{lmDTC}$ and changes in kinematic variables over time were found between concussed and healthy controls. Additionally, despite the small sample, this presents the first investigation into turning kinematics post-concussion and provides the only longitudinal data spanning up to one year. Variations in a few data points, such as the one-year follow-up data among controls, likely had particularly strong influence on the recovery timeline, potentially leading to over- or 
underestimated recovery in some instances (e.g., for $\theta_{2}$ the recovery time may have been underestimated given the decrease in inclinations of the two controls subjects at the one-year follow-up).

Another limitation was the potential variation of cognitive task performance. The serial seven subtraction task was chosen based on its complexity and the ability to discriminate concussed and healthy individuals [23]. While participants were directed to subtract as fast as possible, it was difficult to discern whether priority was given to the cognitive or motor task, as the response rate and number of errors were difficult to characterize. However, because this investigation was concerned with the kinematic deficits during locomotion that manifest in asymptomatic cleared athletes, potential differences in prioritization do not change the present conclusions about diminished dual-task capacity. Similar prioritization would likely occur during real-life situations outside the testing environment.

Finally, the use of a small set of reflective markers may have introduced small error into the $\mathrm{COM}_{\mathrm{UB}}$ and inclination angle $\theta$ calculations compared to a full body marker set. Other markers were placed on the participants, but proved unreliable due to the testing environment.

\section{Conclusions}

Turning gait kinematics changed differently over time between concussed and healthy athletes, suggesting that turning locomotion recovers slower than clinical signs or symptoms. These results also suggest that turning velocity and stride time may be useful clinical assessment tools and that turning mechanics post-concussion, reflected in the mediolateral inclination angles during turning gait, may reflect potential kinetic differences during sportrelated maneuvers (e.g., cutting) that could contribute to the higher musculoskeletal injury rates post-concussion $[52,54]$. Turning gait and change of direction tasks are recommended for inclusion in future locomotor studies of concussion-related kinematic differences and injury potential [16], and that such studies extend for six months to one year post-concussion to capture the full recovery timeline. Overall, these results reaffirm that concussions can present persistent gait modifications, and that when and how such gait disturbances subside should be an important area for future research.

\section{Additional file}

Additional file 1: Dataset used for all analysis. (XLSX $400 \mathrm{~kb}$ )

\section{Abbreviations}

$A$, power law gain factor between velocity and radius of curvature; $\mathrm{BOS}$, base of support; C, concussed athletes; CDTC, cognitive dual-task cost; COM, whole-body center of mass; $\mathrm{COM}_{\cup \mathrm{B}}$, center of mass of the upper body; DT, dual-task; DTC, dual-task cost; $\mathrm{H}$, healthy matched controls; ImDTC, locomotor dual-task cost; $\mathrm{ML}$, mediolateral; $\mathrm{mTBI}$, mild traumatic brain injury; NCAA, National Collegiate Athletic Association; RTP, return to play; SD, standard deviation; SE, standard error; ST, single-task; $a$, power law exponent between velocity and radius of curvature; $\mu$, coefficient of friction

\section{Acknowledgements}

The authors would like to thank Dr. Thurmon Lockhart for his general support during the design of the study. The authors would also like to thank Lauren Becker and Brett Griesemer for assisting with data collection, and Nora Fino for statistical consultation.

\section{Funding}

PCF was supported by an NSF Graduate Research Fellowship (Grant No. DGE0822220). Opinions and findings do not represent the views of the NSF. Additional support for publication was provided by Virginia Tech's Open Access Subvention Fund.

\section{Availability of data and materials}

The dataset supporting the conclusions of this article is included within the article and within Additional file 1.

\section{Authors' contributions}

PCF and PGB contributed to the conceptualization and design of the study. PCF contributed to the data acquisition and analysis. PCF, MAN, and PGB contributed to the interpretation of the data and the writing and revisions. All authors read and approved the final manuscript.

\section{Competing interests}

The authors declare that they have no competing interests.

\section{Consent for publication}

Written informed consent for publication of de-identified data and/or images was obtained from all participants. A copy of the consent form is available for review by the Editor of this journal.

\section{Ethics approval and consent to participate}

All protocols and procedures were approved by the Virginia Tech Institutional Review Board (IRB\# 14-677), and all participants gave informed written consent.

\section{Author details}

${ }^{1}$ Department of Neurology, Oregon Health \& Science University, 3181 SW Sam Jackson Park Road, L226, Portland, OR 97239, USA. ²Department of Mechanical Engineering, Virginia Tech, Blacksburg, USA. ${ }^{3}$ Department of Industrial and Systems Engineering, Virginia Tech, Blacksburg, USA. ${ }^{4}$ Edward Via College of Osteopathic Medicine, Blacksburg, USA.

Received: 3 March 2016 Accepted: 17 July 2016

Published online: 25 July 2016

\section{References}

1. Langlois JA, Rutland-Brown W, Wald MM. The epidemiology and impact of traumatic brain injury: a brief overview. J Head Trauma Rehabil. 2006;21:375-8.

2. Daneshvar DH, Nowinski CJ, McKee A, Cantu RC. The epidemiology of sportrelated concussion. Clin Sports Med. 2011;30:1

3. McCrory P, Meeuwisse WH, Aubry M, Cantu B, Dvořák J, Echemendia RJ, Engebretsen L, Johnston K, Kutcher JS, Raftery M. Consensus statement on concussion in sport: the 4th International Conference on Concussion in Sport held in Zurich, November 2012. Br J Sports Med. 2013;47:250-8.

4. Slobounov S, Gay M, Johnson B, Zhang K. Concussion in athletics: ongoing clinical and brain imaging research controversies. Brain Imaging Behav. 2012;6:224-43.

5. Johnson B, Zhang K, Gay M, Horovitz S, Hallett M, Sebastianelli W, Slobounov S. Alteration of brain default network in subacute phase of injury in concussed individuals: resting-state fMRI study. Neuroimage. 2012;59:511-8.

6. Slobounov SM, Zhang K, Pennell D, Ray W, Johnson B, Sebastianelli W. Functional abnormalities in normally appearing athletes following mild traumatic brain injury: a functional MRI study. Exp Brain Res. 2010;202:341-54. 
7. Baker CS, Cinelli ME. Visuomotor deficits during locomotion in previously concussed athletes 30 or more days following return to play. Physiol Rep. 2014;2. doi: 10.14814/phy2.12252

8. Buckley TA, Munkasy BA, Tapia-Lovler TG, Wikstrom EA. Altered gait termination strategies following a concussion. Gait Posture. 2013;38:549-51.

9. Catena RD, van Donkelaar P, Chou LS. Different gait tasks distinguish immediate vs. long-term effects of concussion on balance control. J Neuroeng Rehabil. 2009;6:25.

10. Cossette I, Ouellet MC, McFadyen BJ. A preliminary study to identify locomotor-cognitive dual tasks that reveal persistent executive dysfunction after mild traumatic brain injury. Arch Phys Med Rehabil. 2014;95:1594-7.

11. Fait P, Swaine B, Cantin J-F, Leblond J, MCFadyen BJ. Altered integrated locomotor and cognitive function in elite athletes 30 days post concussion: A preliminary study. J Head Trauma Rehabil. 2013;28:293-301.

12. Howell DR, Osternig LR, Chou L-S. Dual-task effect on gait balance control in adolescents with concussion. Arch Phys Med Rehabil. 2013;94:1513-20.

13. Martini DN, Sabin MJ, DePesa SA, Leal EW, Negrete TN, Sosnoff J, Broglio SP. The chronic effects of concussion on gait. Arch Phys Med Rehabil. 2011:92:585-9.

14. Niechwiej-Szwedo E, Inness EL, Howe JA, Jaglal S, Mcllroy WE, Verrier MC. Changes in gait variability during different challenges to mobility in patients with traumatic brain injury. Gait Posture. 2007;25:70-7.

15. Parker TM, Osternig LR, Van Donkelaar P, Chou L. Gait stability following concussion. Med Sci Sports Exerc. 2006;38:1032.

16. Powers KC, Kalmar JM, Cinelli ME. Dynamic stability and steering control following a sport-induced concussion. Gait Posture. 2014;39:728-32.

17. Register-Mihalik J, Littleton A, Guskiewicz K. Are divided attention tasks useful in the assessment and management of sport-related concussion? Neuropsychol Rev. 2013;23:300-13.

18. Yogev-Seligmann G, Hausdorff JM, Giladi N. The role of executive function and attention in gait. Mov Disord. 2008;23:329-42.

19. Grabiner MD, Troy KL. Attention demanding tasks during treadmill walking reduce step width variability in young adults. J Neuroeng Rehabil. 2005;2:25

20. Catena RD, van Donkelaar P, Chou L-S. Cognitive task effects on gait stability following concussion. Exp Brain Res. 2007;176:23-31.

21. Catena RD, van Donkelaar P, Chou L-S. The effects of attention capacity on dynamic balance control following concussion. J Neuroeng Rehabil. 2011;8:8.

22. Fait $\mathrm{P}, \mathrm{McF}$ adyen $\mathrm{B}, \mathrm{S}$ waine $\mathrm{B}, \mathrm{Cantin} \mathrm{J}$. Alterations to locomotor navigation in a complex environment at 7 and 30 days following a concussion in an elite athlete. Brain Inj. 2009;23:362-9.

23. Howell D, Osternig L, Koester M, Chou L-S. The effect of cognitive task complexity on gait stability in adolescents following concussion. Exp Brain Res. 2014;232:1773-82.

24. Lee H, Sullivan SJ, Schneiders AG. The use of the dual-task paradigm in detecting gait performance deficits following a sports-related concussion: a systematic review and meta-analysis. J Sci Med Sport. 2013;16:2-7.

25. Tenenbaum G, Bar-Eli M. Decision making in sport: a cognitive perspective. In: Handbook of research on sport psychology New York. 1993. p. 171-92.

26. Reed N, Fait P, Zabjek K, McFadyen B, Taha T, Keightley M. Concussion and concurrent cognitive and sport-specific task performance in youth ice hockey players: a single-case pilot study. J Neurol Neurophysiol. 2013;4:171. doi:10.4172/2155-9562.1000171

27. Chiu S-L, Osternig L, Chou L-S. Concussion induces gait inter-joint coordination variability under conditions of divided attention and obstacle crossing. Gait Posture. 2013;38:717-22.

28. Chou L-S, Kaufman KR, Walker-Rabatin AE, Brey RH, Basford JR. Dynamic instability during obstacle crossing following traumatic brain injury. Gait Posture. 2004;20:245-54.

29. Glaister BC, Bernatz GC, Klute GK, Orendurff MS. Video task analysis of turning during activities of daily living. Gait Posture. 2007;25:289-94.

30. Takei Y, Grasso R, Berthoz A. Quantitative analysis of human walking trajectory on a circular path in darkness. Brain Res Bull. 1996;40:491-5.

31. Courtine G, Papaxanthis C, Schieppati M. Coordinated modulation of locomotor muscle synergies constructs straight-ahead and curvilinear walking in humans. Exp Brain Res. 2006;170:320-35.

32. Courtine G, Schieppati M. Human walking along a curved path. I. Body trajectory, segment orientation and the effect of vision. Eur J Neurosci. 2003;18:177-90.

33. Courtine G, Schieppati M. Human walking along a curved path. II. Gait features and EMG patterns. Eur J Neurosci. 2003;18:191-205.
34. Fino $P$, Lockhart $T$. Required coefficient of friction during turning at selfselected slow, normal, and fast walking speeds. J Biomech. 2014;47:1395-400.

35. Yamaguchi $T$, Yano M, Onodera H, Hokkirigawa K. Kinematics of center of mass and center of pressure predict friction requirement at shoe-floor interface during walking. Gait Posture. 2012;38:209-14.

36. Yamaguchi $\mathrm{T}$, Yano $\mathrm{M}$, Onodera $\mathrm{H}$, Hokkirigawa K. Effect of turning angle on falls caused by induced slips during turning. J Biomech. 2012;45:2624-9.

37. Fino PC, Lockhart TE, Fino NF. Corner height influences center of mass kinematics and path trajectory during turning. J Biomech. 2015;48:104-12.

38. Taylor M, Dabnichki P, Strike S. A three-dimensional biomechanical comparison between turning strategies during the stance phase of walking. Hum Mov Sci. 2005;24:558-73.

39. De Leva P. Adjustments to Zatsiorsky-Seluyanov's segment inertia parameters. J Biomech. 1996;29:1223-30.

40. Huxham F, Gong J, Baker R, Morris M, lansek R. Defining spatial parameters for non-linear walking. Gait Posture. 2006;23:159-63.

41. Hicheur H, Vieilledent S, Richardson M, Flash T, Berthoz A. Velocity and curvature in human locomotion along complex curved paths: a comparison with hand movements. Exp Brain Res. 2005;162:145-54.

42. Olivier A-H, Cretual A. Velocity/curvature relations along a single turn in human locomotion. Neurosci Lett. 2007:412:148-53.

43. Viviani P, Cenzato M. Segmentation and coupling in complex movements. J Exp Psychol Hum Percept Perform. 1985;11:828.

44. Glaister BC, Orendurff MS, Schoen JA, Klute GK. Rotating horizontal ground reaction forces to the body path of progression. J Biomech. 2007:40:3527-32.

45. Pham QC, Hicheur H, Arechavaleta G, Laumond JP, Berthoz A. The formation of trajectories during goal-oriented locomotion in humans. II. A maximum smoothness model. Eur J Neurosci. 2007;26:2391-403.

46. Fino PC. A preliminary study of longitudinal differences in local dynamic stability between recently concussed and healthy athletes during single and dual-task gait. J Biomech. 2016:49:1983.

47. Hicheur H, Pham QC, Arechavaleta G, Laumond JP, Berthoz A. The formation of trajectories during goal-oriented locomotion in humans. I. A stereotyped behaviour. Eur J Neurosci. 2007;26:2376-90.

48. Sreenivasa MN, Frissen I, Souman JL, Ernst MO. Walking along curved paths of different angles: the relationship between head and trunk turning. Exp Brain Res. 2008:191:313-20.

49. Catena RD, van Donkelaar P, Chou L-S. Altered balance control following concussion is better detected with an attention test during gait. Gait Posture. 2007;25:406-11

50. McFadyen BJ, Swaine B, Dumas D, Durand A. Residual effects of a traumatic brain injury on locomotor capacity: a first study of spatiotemporal patterns during unobstructed and obstructed walking. J Head Trauma Rehabil. 2003:18:512-25.

51. Hewett TE, Torg JS, Boden BP. Video analysis of trunk and knee motion during non-contact anterior cruciate ligament injury in female athletes: lateral trunk and knee abduction motion are combined components of the injury mechanism. Br J Sports Med. 2009;43:417-22.

52. Lynall RC, Mauntel TC, Padua DA, Mihalik JP. Acute lower extremity injury rates increase following concussion in College Athletes. Med Sci Sports Exerc. 2015;47:2487.

53. Brooks MA, Peterson K, Biese K, Sanfilippo J, Heiderscheit BC, Bell DR. Concussion Increases Odds of Sustaining a Lower Extremity Musculoskeletal Injury After Return to Play Among Collegiate Athletes. Am J Sports Med. 2016:44:742-7.

54. Nordström A, Nordström P, Ekstrand J. Sports-related concussion increases the risk of subsequent injury by about $50 \%$ in elite male football players. $\mathrm{Br}$ J Sports Med. 2014;48:1447-50. 